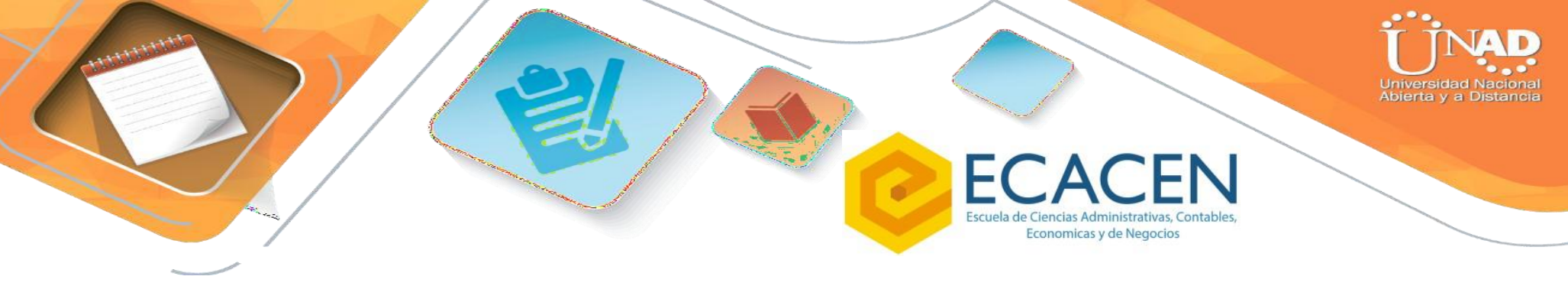

\title{
CARACTERIZACIÓN DE LA NORMAS INTERNACIONALES DE AUDITORIA (NIAS) DIRECCIONADA AL EFECTIVO Y EQUIVALENTES DEL EFECTIVO EN LA EMPRESA DE SERVICIOS PÚBLICOS EMPITALITO E.S.P UBICADA EN EL MUNICIPIO DE PITALITO HUILA
}

\author{
CHARACTERIZATION OF THE INTERNATIONAL AUDITING \\ STANDARDS (NIAS) DIRECTED TO CASH AND CASH \\ EQUIVALENTS IN THE PUBLIC SERVICES COMPANY \\ EMPITALITO E.S.P LOCATED IN THE MUNICIPALITY OF \\ PITALITO HUILA
}

\section{Autor 1}

Mercedes Cruz Zúñiga

Contador Público, Estudiante de la Maestría Administración de

Organizaciones, Docente de MT de la Escuela ECACEN, CCAV Pitalito. Google Académico:

https://scholar.google.es/citations?view_op=new_profile\&hl=es

pertenezco al Grupo Cananguchales, semillero YUMA.

ORCID: https://orcid.org/my-orcid

Correo electrónico: mercedes.cruz@unad.edu.co

\section{Autor 2}

Sindy Julieth Barrera Suarez

Contador Público, Master en dirección estratégica, Docente de MT de la Escuela ECACEN, CCAV Pitalito. Google Académico:

https://scholar.google.es/citations?view_op=new_profile\&hl=es

pertenezco al Grupo Cananguchales, semillero YUMA.

ORCID: https://orcid.org/0000-0003-3750-8679

Correo electrónico: sindy.barrera@unad.edu.co

\section{RESUMEN}

Las Normas Internacionales de Auditoria (NIAs) emitidas por Comité Internacional de Normas de Auditoría y Seguridad IAASB de la Federación Internacional de Contadores (IFAC) expedidas decreto 302 del 2015. Anteriormente llamadas Normas de Auditoria Generalmente aceptadas (NAGAS) siguiendo los elementos fundamentales para identificar y evaluar 


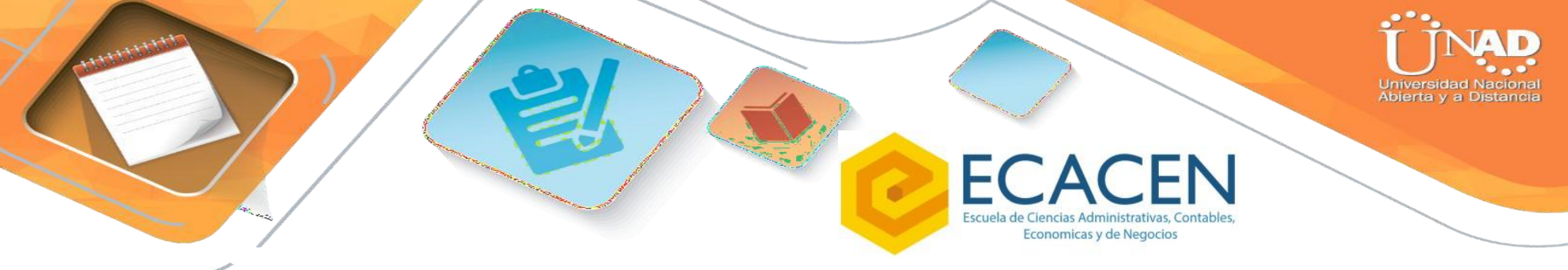

This research is of a mixed approach, qualitative and quantitative data will be linked, and the results generated will serve as a starting point for the accounting profession through strategies that facilitate the understanding of the (NIAs). Because of the foregoing, it will provide strategies that will minimize physical and emotional wear and tear on public accountants who find it difficult to apply and understand them and therefore improve the productivity of companies that triggers changing processes and procedures due to globalization.

Keywords: Audit; NIAS standards; Cash Equivalent to Cash; Characterization; Public Services Company.

\section{INTRODUCCIÓN}

Lo que originó la presente investigación es la preocupación por el desconocimiento de las (NIAs), como también la continuidad que se le da en la aplicabilidad de las (NIAs) en Empitalito E.S.P; ya que es una empresa del sector público, que cada cuatro años cambia de Gerente y de igual manera los profesionales que hacen parte de la estructura contable, financiera y presupuestal, como también su control interno y demás departamentos de libre nombramiento y remoción, razones por las cuales se busca establecer cuál es el método óptimo en la implementación de las (NIAs) identificando los factores que debemos evaluar en un sector específico de la empresa de Empitalito. Lo que se va a demostrar con la exploración de la presente investigación es replicar a los Contadores, Auditores y Revisores Fiscales el método eficiente a la hora de implementar la norma direccionada específicamente en efectivo y equivalentes del efectivo.

La finalidad del Objetivo General es Caracterizar las Normas Internacionales de Auditoria (NIAs) direccionada al efectivo y equivalentes del efectivo en la empresa de servicios públicos Empitalito E.S.P ubicada en el Municipio de Pitalito Huila. Las aspiraciones o propósitos que se pretende lograr con los Objetivos Específicos son los que detallan la completa realización de las (NIAs) como: 1. Identificar las Normas Internacionales de Auditoria (NIAs) direccionadas al efectivo y equivalentes del efectivo en las empresas de servicios públicos. 2. Seleccionar la actividad técnica principal respecto a la operación de la empresa Empitalito. (Acueducto, alcantarillado y aseo). La información 


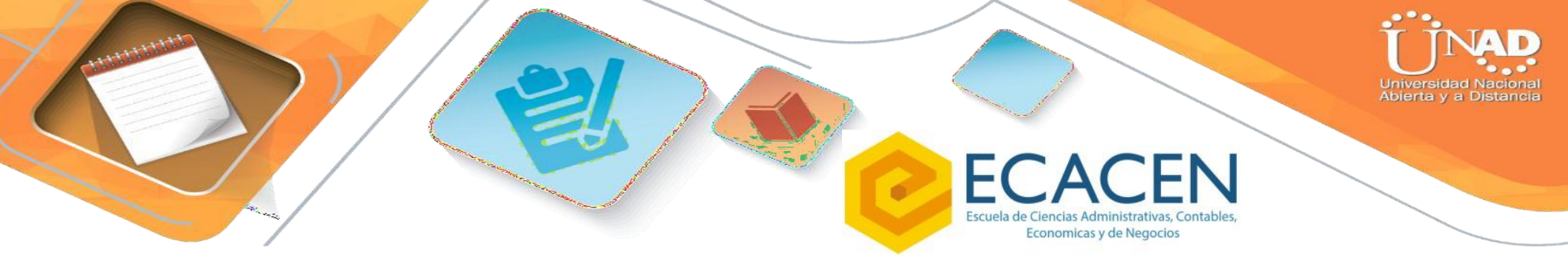

financiera alcanza el grado de confiabilidad para que las empresas extranjeras inviertan en nuestro municipio y alcancen la transparencia, relevancia, comparabilidad y sobre todo razonabilidad en la información financiera.

\section{METODOLOGÍA}

Las Normas de Auditoria (NIAs) direccionada al efectivo y equivalentes del efectivo en la empresa de servicios públicos Empitalito ubicada en el municipio de Pitalito Huila, inicialmente se realizará una revisión documental bibliográfica y de la entidad, y se aplicaran como instrumentos de investigación una encuesta y una entrevista semiestructurada focalizada a encontrar las posibles falencias y no aplicabilidad de la norma en dicha empresa.

Población: EMPITALITO ESP empresa de servicios públicos domiciliarios de acueducto, alcantarillado y aseo en el municipio de Pitalito (Huila).

Muestra: Sistema de acueducto direccionado a la partida contable del efectivo y equivalentes del efectivo de la empresa de servicios públicos domiciliarios de Pitalito EMPITALITO ESP.

\section{Fases de la metodología:}

\section{Fase 1}

Identificar las Normas Internacionales de Auditoria (NIAs) direccionadas al efectivo y equivalentes del efectivo en las empresas de servicios públicos.

Tipo de Investigación:

Documental Población:

Empitalito ESP

Tipo de información: primaria y secundaria

\section{Fase 2}

Seleccionar la actividad técnica principal respecto a la operación de la empresa Empitalito. (Acueducto, alcantarillado y aseo).

Tipo de Investigación:

descriptiva Población:

Empitalito ESP 


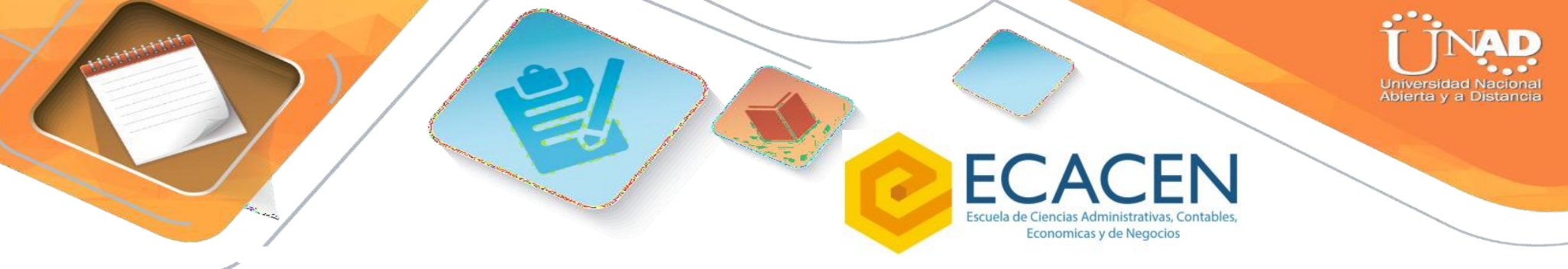

Internacionales de Información Financiera (NIIF) Y Normas Internacionales de Auditoria (NIA).

Para realizar el proceso de caracterización de la Empresa de Servicios Públicos de Pitalito, es importante seleccionar la actividad técnica principal de acuerdo a su operación (Acueducto, Alcantarillado, Aseo y Alumbrado Público).

\section{Actividad técnica (Acueducto)}

Cuenta con una planta de tratamiento la cual está ubicada en la zona urbana, captando las aguas del rio Guachicos, este servicio se presta a los usuarios del Municipio en la Zona Urbana aproximadamente al 99\% del total de la población; mediante Resolución 1096 de 2015 cuenta una concesión de aguas superficiales por 10 años, en ella autoriza una captación de 668.92LPS.

\section{Actividad técnica (Alcantarillado)}

Empitalito realiza recolección y transporte de las aguas residuales, con esto da cumplimiento a los requisitos legales, aprobada por la CAM mediante la resolución 3037 de 2008 y reformulado mediante Res. CAM 2729 DE 2011 el servicio se presta en la parte urbana. Lo que se consume de acueducto se tiene en cuenta un $85 \%$ que se cobra por el Servicio de Alcantarillado, este se presta aproximadamente el $98 \%$ de los usuarios.

\section{Actividad técnica (Aseo)}

Este servicio es prestado en la zona urbana y en algunos sectores rurales; además, realiza barrido de vías y limpieza.

El tratamiento de los residuos sólidos y disposición final, lo realiza La Empresa Biorgánicos del sur del Huila, mediante contratos interadministrativos con EMPITALITO.

\section{Alumbrado público}

Este servicio se presta mediante convenios interadministrativos con el municipio de Pitalito, tanto en la zona urbana como rural, reparando las averías e incidencias del sistema de Alumbrado Público. 


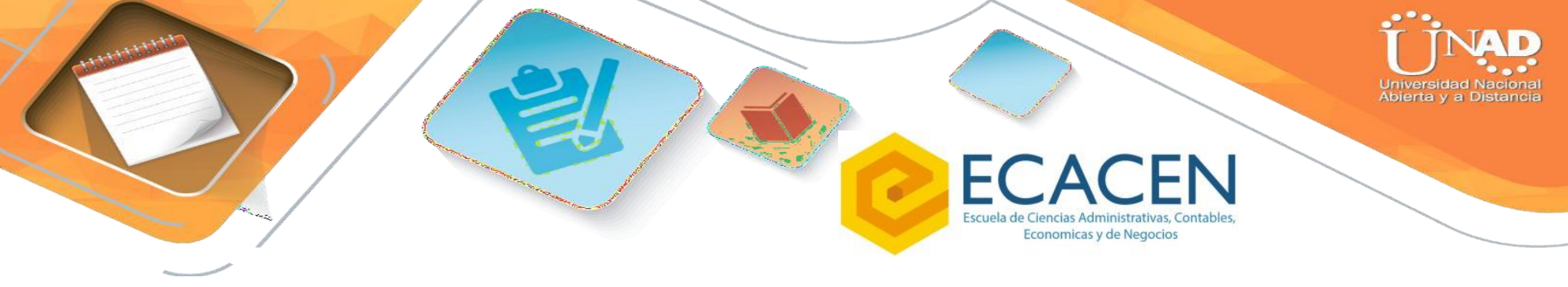

\section{Área Administrativa}

EMPITALITO cuenta con la oficina Operativa, la cual maneja los servicios de Acueducto, Alcantarillado y Aseo; a su vez cada uno de los servicios cuenta con el personal para atender los requerimientos necesarios para mantener un óptimo servicio.

La empresa maneja un Software contable, el cual tiene diferentes módulos, los cuales funcionan en la oficina de presupuesto, tesorería, almacén y facturación, los funcionarios encargados alimentan el Software y está información es conciliada con en la oficina de contabilidad, es donde llega toda la información.

El proceso contable empieza en facturación, donde se realizan las lecturas del consumo mensual de cada uno de los usuarios; para emitir la factura, al ser cancelada por el usuario, se realiza la recepción de los recibos en la oficina de tesorería y luego a facturación, quedando en el software contable los ingresos por parte de la oficina de tesorería y las cuentas por cobrar emitidas por facturación.

En cuanto a los costos incurridos para prestar estos servicios el trámite empieza por la necesidad emitida por la Unidad Operativa, luego contratación realiza el proceso legal, incluyendo la disponibilidad presupuesta dada por presupuesto; el almacén se encarga de las compras, y todas estas se van registrando en la contabilidad, debido a que el almacén tiene un módulo contable el cual va alimentando la contabilidad de la empresa, luego todos los soportes pasan a contabilidad para ser causado y finalmente a tesorería donde se efectúa el pago por canales virtuales.

Con la correcta aplicación de las (NIAs) se fortalecerán las competencias de los estudiantes, contadores públicos. Inicia una nueva concesión del desempeño, el sector empresarial va a tener la capacidad de identificar al ser humano como sujeto productivo, se reduce la incertidumbre a nivel empresarial y estarán enfocados a la producción, competitividad, inversión en la región, eficiencia de los recursos, supervivencia de los mercados, oportunidad de gestión empresarial, evaluación del riesgo y sobre todo a la calidad de la información contable. Además, se ve involucrado el sector educativo donde se busca analizar cada uno de los componentes de las (NIAs), que los Estudiantes se puedan apropiar de los conceptos y tener una concesión más amplia de la profesión. 


\section{ECACEN}

Se evidencia que las (NIAs) es una herramienta de alta calidad que reconoce el proceso de la auditoria y la razonabilidad de los Estados Financieros en efectivo y equivalentes del efectivo en la empresa de servicios públicos Empitalito.

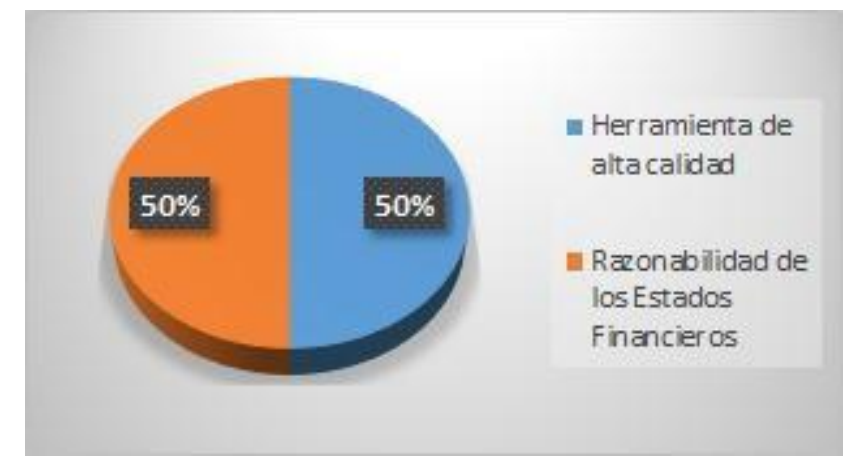

Figura 1. Normas Internacionales de Auditoria.

Fuente: elaboración propia con datos tomados de la presente investigación.

De esta manera unifica los elementos técnicos para la planeación, evaluación del riesgo de detección y de incorrección material. Se observa que el código de ética es la principal reglamentación para los profesionales que ejercen en las empresas de servicios públicos.

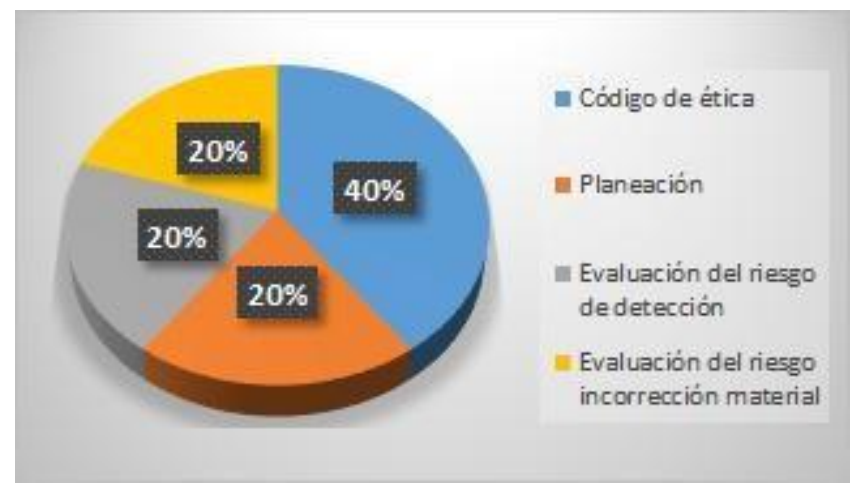

Figura 2. Elementos Técnicos.

Fuente: elaboración propia con datos tomados de la presente investigación. 


\section{ECACEN}

El desarrollo de las nuevas competencias se debe a la aplicación de las (NIAs) por su obligatoriedad de la actualización de conocimientos y además es eficaz y satisfactorio. La aplicación del conjunto de estándares de auditoria demuestra los diferentes retos que se reflejan en la adecuada supervisión, independencia, materialidad y control interno.

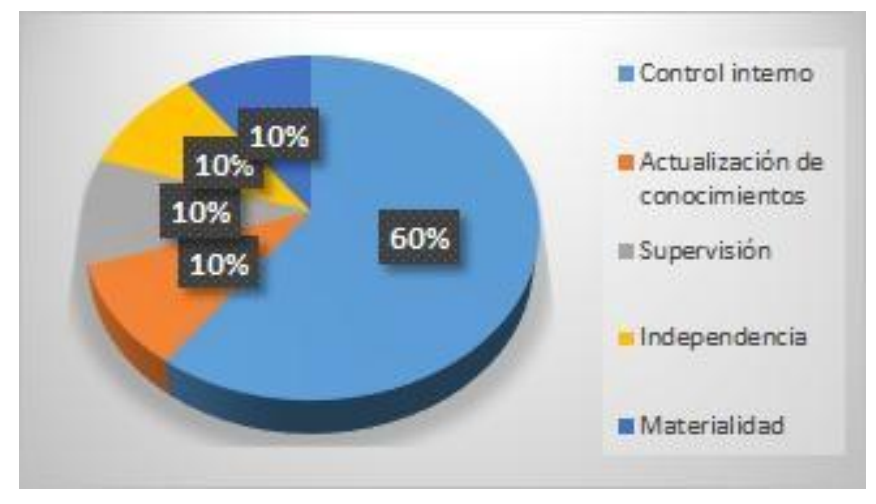

Figura 3. Aplicación conjunta de estándares.

Fuente: elaboración propia con datos tomados de la presente investigación.

Se incluyen las prácticas de revisión, atestación y aseguramiento donde se administra el riesgo. Se demuestra el control del disponible y depósitos, relaciona todos los ciclos transaccionales y se reflejan los saldos importantes del efectivo.

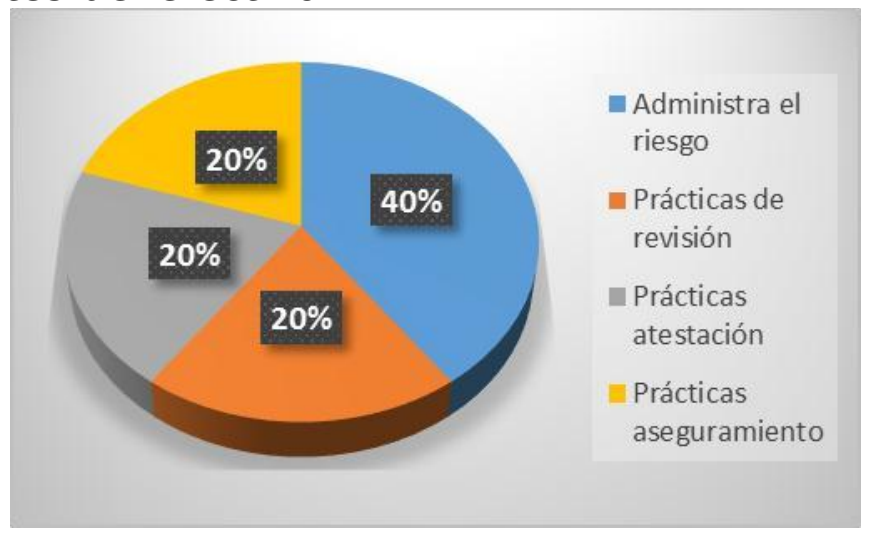

Figura 4. Prácticas de aplicación.

Fuente: elaboración propia con datos tomados de la presente investigación. 


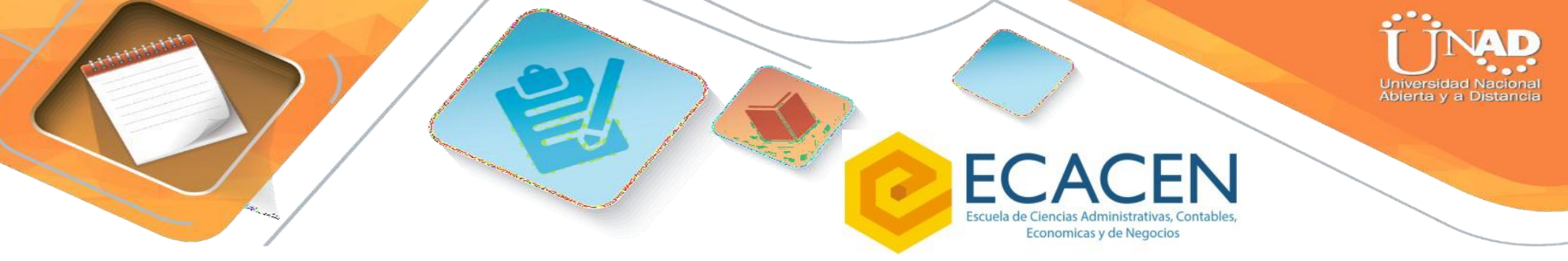

Se tiene en cuenta los (Clientes, Ingresos y Egresos) como también el área de tesorería donde se realizan las operaciones desde los controles directos, controles generales, controles gerenciales y controles independientes desde la prueba, clasificación y confirmación.

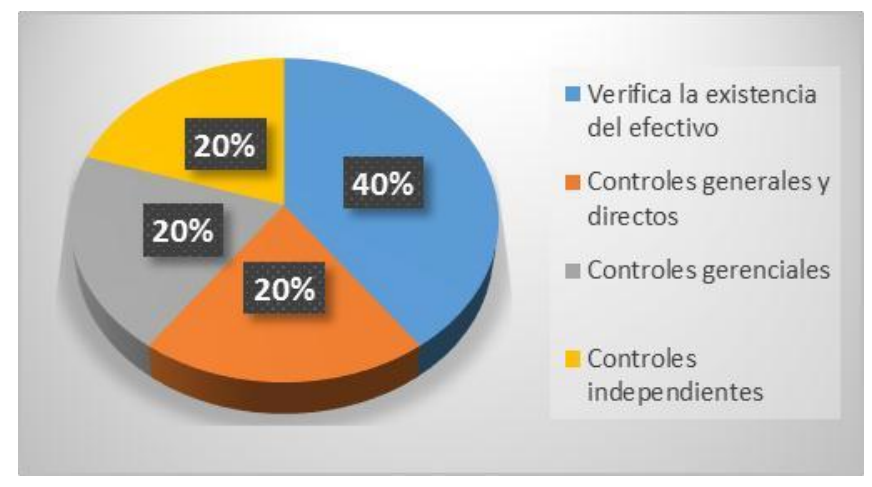

Figura 5. Cuentas representativas.

Fuente: elaboración propia con datos tomados de la presente investigación.

\section{CONCLUSIONES}

Las Normas Internacionales de Auditoria (NIAs) direccionada al efectivo y equivalentes del efectivo en la empresa de servicios públicos Empitalito E.S.P trae consigo considerables efectos como: la mejora en la calidad de las auditorías, la confiabilidad de información financiera, la prevención del fraude, la observancia del código de ética, la idoneidad del auditor como empresario, las responsabilidades de la administración y el impacto en las organizaciones.

Los servicios públicos son de primera necesidad, estos deben ser debidamente auditados; es necesario que el Gerente pueda tomar decisiones teniendo el análisis de la entidad que solo puede ir enmarcado si existe una buena administración financiera organizada y reglamentada. Normalmente el eje estratégico de los servicios público es la parte operativa; sin embargo, se debe saber si es rentable, si los gastos y costos no superan los ingresos, por esto debe tener el control del área financiera.

La vigilancia y control es el proceso que mayor importancia tiene frente 
a la información fraudulenta ya que este sistema maneja 17 variables y 7 están directamente relacionadas con el desorden y malas prácticas administrativas, esto hace que no exista la vulnerabilidad de cada una de las transacciones provenientes de la operación de la empresa. El rol del contador público predomina en mejorar el ejercicio de la práctica desde preparación, actualización permanente de la evolución de los mercados, el conocimiento de los cambios estructurales y entrenamiento para que pueda evaluar las estimaciones complejas como mecanismo de detección desarrollando conciencia de control en todo el proceso de aplicación y cumplimiento estricto de las Normas Internacionales de Auditoria (NIA).

\section{REFERENCIAS}

Baracaldo-Lozano, N. A. (2013). ¿Es garante del principio de transparencia la implementación de normas internacionales en Colombia?

Cuadernos de Contabilidad, 14 (36), 1097-1120.

Bustamante, L. M. O., Londoño, J., Esteban Zamarra, \& Sanabria, J. A. G. (2011).

¿Cuál ha sido la responsabilidad de la auditoría en los escándalos financieros? Contaduría Universidad De Antioquia, (58), 117-145.

https://search.proquest.com/docview/1679860397?accountid= 48797

Cabeza del Salvador, I. (2020). El modelo de informe de auditoría de cuentas en las NIA-ES-SP. Auditoría Pública: Revista de Los Organos Autónomos de Control Externo, 75, 45-58. http://search.ebscohost.com. bibliotecavirtual.unad.edu.co/login.aspx ?direct $=\mathrm{tr}$ ue\&db=edsdnp\&AN=edsdnp. 7444142ART\&lang=es\&site=eds- 
$9826 Z$ Ramírez Cacho, S.I. (2013). El proceso iberoamericano de convergencia con las

normas internacionales de auditoría. Distrito Federal, México: Plaza y Valdés.

http://site.ebrary.com/lib/bibliouniminutosp/detail.action?docID =1125 9233

Salas-Ávila, J. A. \& Reyes-Maldonado, N. M. (2015). Modelo propuesto para la detección de fraudes por parte de los auditores internos basado en las normas internacionales de auditoría. Cuadernos de Contabilidad, 16(42), 579-

623.http://dx.doi.org/10.11144/Javeriana.cc16-42.mpdf

Vega, A. S. (2019). Novedades en las normas internacionales de auditoría (2019-2020). Técnica Contable y Financiera, 22, 2023.

http://search.ebscohost.com.bibliotecavirtual.unad.edu.co/login.aspx ?direct $=$ tr ue $\& d b=$ edsdnp\&AN =edsdnp. 7087753ART\&lang=es\&site=edslive\&scope = site. 